\title{
Computational Prediction of HCV RNA Polymerase Inhibitors from Alkaloid Library
}

\author{
Samuel Olawale Olubode ${ }^{1 * *(D)}$, Olaposi Idowu Omotuyi ${ }^{2}$ (D), David Oluwadare Fadipe ${ }^{3 \text { (D) }}$ \\ 1 Department of Biochemistry, Adekunle Ajasin University, Akungba Akoko, Nigeria.; olubodesamuelolawale@gmail.com \\ (S.O.O.); \\ 2 Department of Biochemistry, Adekunle Ajasin University, Akungba Akoko, Nigeria; olaposi.omotuyi@aaua.edu.ng \\ (O.I.O.); \\ 3 Health Service, Adekunle Ajasin University, Akungba Akoko, Nigeria; david.fadipe@aaua.edu.ng (D.O.F.); \\ * Correspondence: olubodesamuelolawale@gmail.com (S.O.O.);
}

Received: 30.04.2021; Revised: 30.06.2021; Accepted: 5.07.2021; Published: 7.09.2021

\begin{abstract}
Hepatitis C virus (HCV) is a global challenge and the leading cause of chronic liver disease. Approximately $30 \%$ of a patient infected with chronic HCV results into liver cirrhosis. WHO reports that $3 \%$ of the world's population has been infected with $\mathrm{HCV}$, which signifies that about 170 million people are at risk of developing chronic liver diseases globally. Research has shown that HCV NS5B polymerase is one of the six non-structural proteins encoded in the approximately 9600 nucleotide genome of $\mathrm{HCV}$, which plays a vital role in the replication and infection of $\mathrm{HCV}$ virus; therefore, it serves as a target enzyme for antiviral therapy against $\mathrm{HCV}$. In this study, Alkaloids, a group of vital secondary metabolites in plants, with cyclic structures containing nitrogen in a negative oxidation state which is limitedly distributed in a living organism, were mined from an online database and screened computationally using a molecular docking approach to predict its inhibitory potential against the replication of HCV's viral RNA. 259 Alkaloids was retrieved and docked, and it resulted that 3-(4methoxyphenyl)-3-[[2-(4-methoxyphenyl)-1-oxoethyl]amino]propanoic acid, 14-norpseurotin A, and (+)-aplysinilin are predicted to be suitable inhibitors against NS5B through their binding pose and interactions with the amino acid residues at the binding site of NS5B. Additionally, hit compounds from the docking result were further subjected to ADME/Tox screening to predict their drug-likeness characteristics, and 3-(4-methoxyphenyl)-3-[[2-(4-methoxyphenyl)-1-oxoethyl]amino]propanoic acid stands out by showing more drug-like characteristics.
\end{abstract}

Keywords: Hepatitis C; Hepatitis C Virus (HCV); non-structural protein 5B (NS5B); alkaloids; molecular docking.

(C) 2021 by the authors. This article is an open-access article distributed under the terms and conditions of the Creative Commons Attribution (CC BY) license (https://creativecommons.org/licenses/by/4.0/).

\section{Introduction}

Hepatitis $\mathrm{C}$ virus (HCV) is generally known as the leading cause of chronic liver disease, which results in Liver cirrhosis in $20 \%-30 \%$ of patients with a chronic infection after 2 to 3 decades [1]. Hepatocellular carcinoma develops once cirrhosis occurs in $1 \%-4 \%$ of these patients per year [2]. Estimation shows that HCV was attributable to one-third of hepatocellular carcinoma cases globally [3], signifying it as a significant public health problem [4].

WHO reports that $3 \%$ of the world's population has been infected with $\mathrm{HCV}$, which signifies that about 170 million people are at risk of developing chronic liver diseases globally. The prevalence of HCV estimated in economically developed countries is comparatively low with $1 \%-2 \%$ population of the adult whereas $5 \%-10 \%$ in less developed countries [4]. HCV 
prevalence is high in people who inject drugs because of the high risk of liver morbidity and mortality [5]. The countries reported with higher prevalence were found in Africa, South-East Asia, Eastern Mediterranean, and the West Pacific, while North America, northern and western Europe, and Australia are areas with lower prevalence [4]. Therefore a pharmacological solution is needed.

Currently, there is no vaccine available to prevent hepatitis $\mathrm{C}$; however, chronic $\mathrm{HCV}$ can be treated and cured. Cirrhosis, the prominent cause of liver cancer, can be caused by untreated HCV [6].

Research has shown that HCV NS5B polymerase is one of the six non-structural proteins that are encoded in the approximately 9600 nucleotide genome of $\mathrm{HCV}$, which plays a vital role in the replication and infection of the HCV virus [7]; HCV NS5B polymerase looks like a right hand topologically in a three-dimensional confirmation that includes: the palm, the finger and the thumb region. The thumb region carries the active site of HCV NS5B polymerase [8]. Therefore it serves as a target enzyme for antiviral therapy against $\operatorname{HCV}[9,10]$. Several inhibitors have been reported in many research works lately against HCV NS5B [11].

This work focuses on predicting the best inhibitor for HCV NS5B from the alkaloid library to inhibit its action using computational tools.

Alkaloids are cyclic compounds containing nitrogen in a negative oxidation state limitedly distributed in living organisms; they are a group of vital secondary metabolites in plants $[12,13]$. Many alkaloids are toxic, and plants use them to protect themselves against violence from other parasitic organisms [14]. Many alkaloids have been used in medicine for several hundred years and even today's prominent drugs [15]. Alkaloids from plant extracts have served as ingredients in poisons and liquid medicine used by ancient people to treat many ailments such as fever, snakebite, and insanity [15]. It has also served as an effective source for drug discovery [16]. Its therapeutic effect on hepatocellular carcinoma, cancer, and diabetes has also been reported [16-18].

Molecular docking is used to accomplish this work. Molecular docking is a field that studies how small molecules (ligand) interact with the binding site of a target protein [19]. Molecular docking is the most used molecular modeling method [20]. The methods employed to accomplish this work include; ligand library generation from online database and preparation, target protein retrieval from online database and preparation, receptor grid generation, docking, and ADMET/Tox Screening of hit compound from alkaloid library.

\section{Materials and Methods}

\subsection{Ligand library generation and preparation.}

Structures of Alkaloid compounds (two dimensional) were mined from Pubchem online database [21] in sdf format and was prepared using ligprep tool [22] (Using Epik [23, 24] at pH 7.0 with OPLS3 [25] force field). Maestro, Schrodinger Suite 2017 [26].

\subsection{Target retrieval and preparation.}

X-ray crystallographic structure of human HCV NS5B complexed with an inhibitor as a co-crystallized ligand at the active site (RNA 960) (PDB ID: 2HWH) [27] was retrieved from Protein Data Bank [28]. It was selected due to the presence of an inhibitor at the active site of the protein [29]. 
The retrieved protein was viewed and prepared using Maestro 11.1 [26], Protein preparation wizard, optimized at $\mathrm{pH}$ 7.0, and minimized using OPLS3 as a force field.

\subsection{Receptor grid generation.}

The receptor grid shows the area between the ligand and the protein where interaction takes place.

The prepared protein grid was generated on the active site through the Receptor Grid Generation tool at the sizes $8.75,34.05$, and $72.92 \mathrm{~A}^{\circ}(\mathrm{X}, \mathrm{Y}$, and $\mathrm{Z})$ to consist of all amino acids at the active site of the protein.

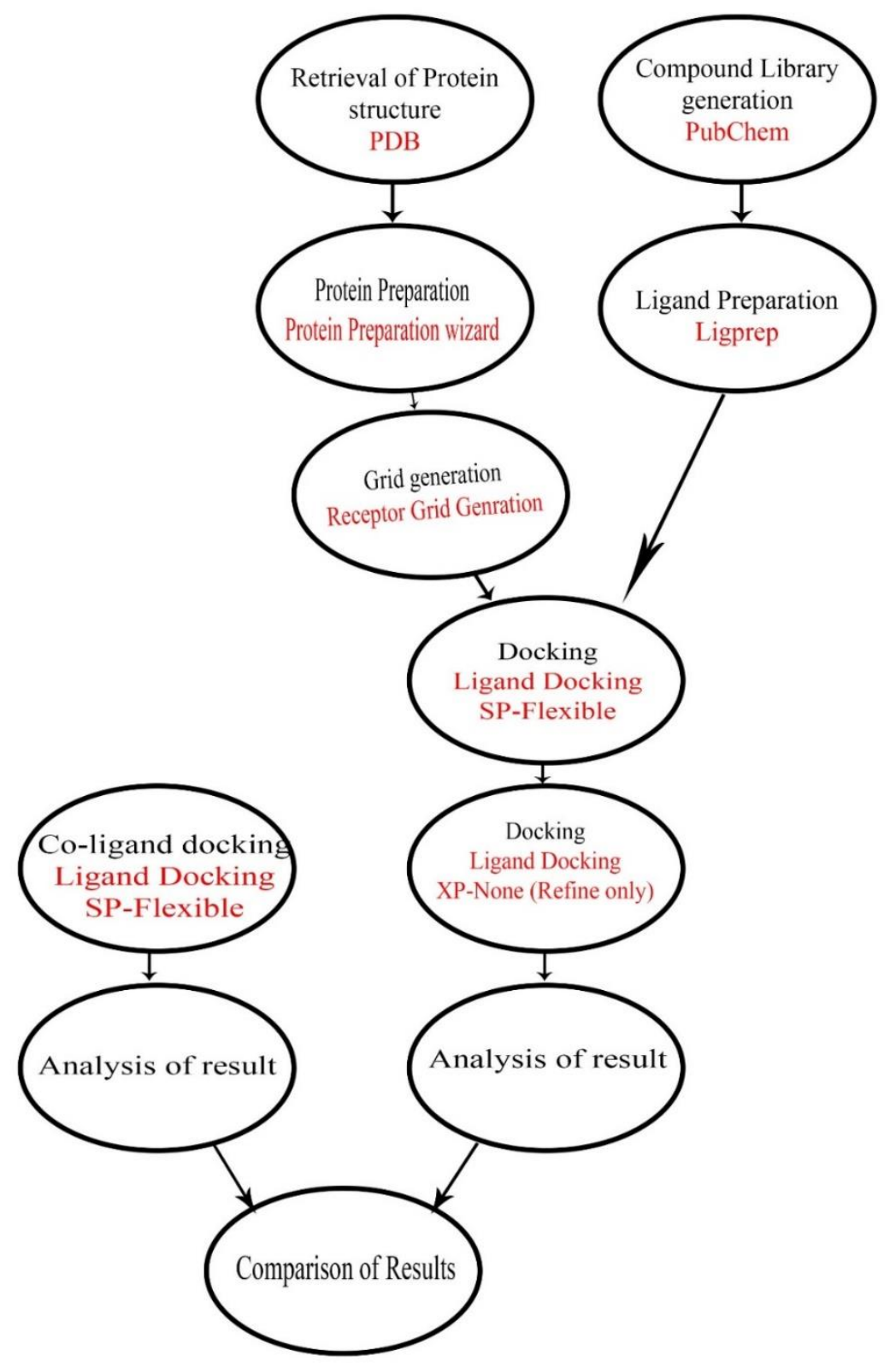

Figure 1. Flow chart of the experimental procedure.

\subsection{Docking.}

The Docking analysis was accomplished using the Glide tool [30, 31] on maestro 11.5 [26]. The molecular docking experiment was carried out with the enzyme-treated as a rigid body, while the ligand's rotatable bonds were set to be flexible. The prepared ligands were 
docked to the prepared protein grid using the ligand docking tools, setting the precision to standard precision (SP) and ligand sampling to rank the ligands according to their docking score flexibly. Docked ligands were re-docked and subjected to extra precision (XP) and the ligand sampling to None (refine only) to refine the compounds and predict the compound with the best interaction with the amino acids in the active site predict its inhibitory action.

HCV NS5B co-crystallized ligand was also docked using the same procedures stated above. The interaction with HCV NS5B was compared with the best inhibitory compounds from the alkaloid library.

\subsection{ADMET/Tox screening.}

To predict drug-like characteristics of Hit compound, from docking, they were further subjected to Absorption, Distribution, Metabolism, Excretion and Toxicity screening (ADMET/Tox Screening) using the Qikproptool. [32]. The rules are valid when; mol. MW < $500, \mathrm{QP} \log \mathrm{Po} / \mathrm{w}<5$, donor $\mathrm{HB} \leq 5$, acceptor $\mathrm{HB} \leq 10$. Maximum is 4; HOA: Human Oral Absorption [33].

\section{Results and Discussion}

\subsection{Result.}

This work shows a computational approach using a molecular docking tool to reveal the molecular interaction, binding pose, and inhibitory potentials of the three (3) best alkaloid compounds compared with the co-crystalized ligand, which was also screened computationally against NS5B. The whole experiment shows that 3 compounds, (3-(4-methoxyphenyl)-3-[[2(4-methoxyphenyl)-1-oxoethyl]amino]propanoic acid, 14-norpseurotin A and (+)-aplysinilin) whose structures are shown in Figure 2, 3, and 4 respectively, shows the best docking score compared to the co-crystallized ligand shown in figure 5. The docking result, including the ADME/Tox screening result, is shown in Table 1, the $2 \mathrm{D}$ interaction of hit compounds compared to the co-crystallized ligand as shown in figure 10. The 3D interaction of these hit compounds within the active site of NS5B compared to the co-crystallized ligand is shown in figure 6 , figure 7 , figure 8 , and figure 9 , respectively.

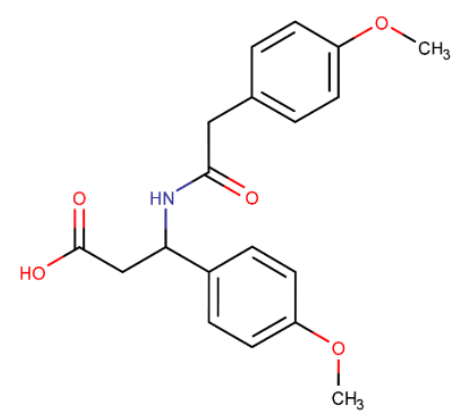

Figure 2. 2d structure of 3-(4-methoxyphenyl)-3-[[2-(4-methoxyphenyl)-1-oxoethyl]amino]propanoic acid. 


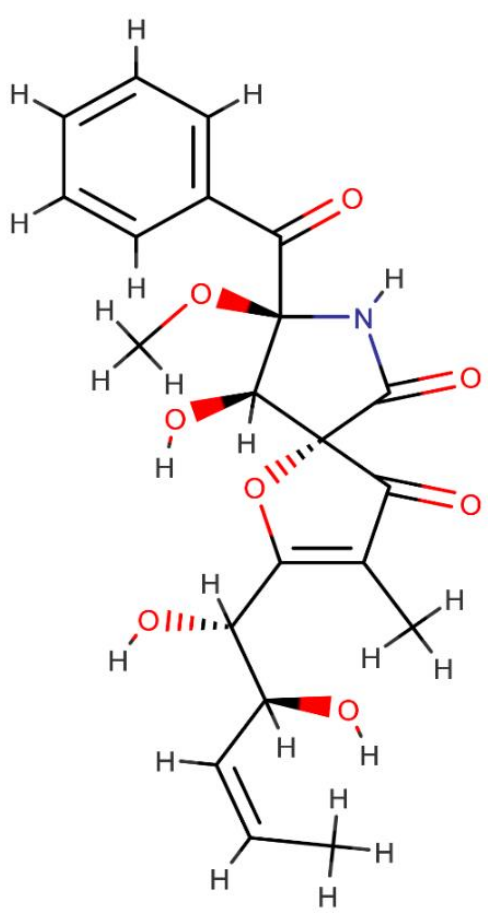

Figure 3. 2d structure of 14-norpseurotin A.

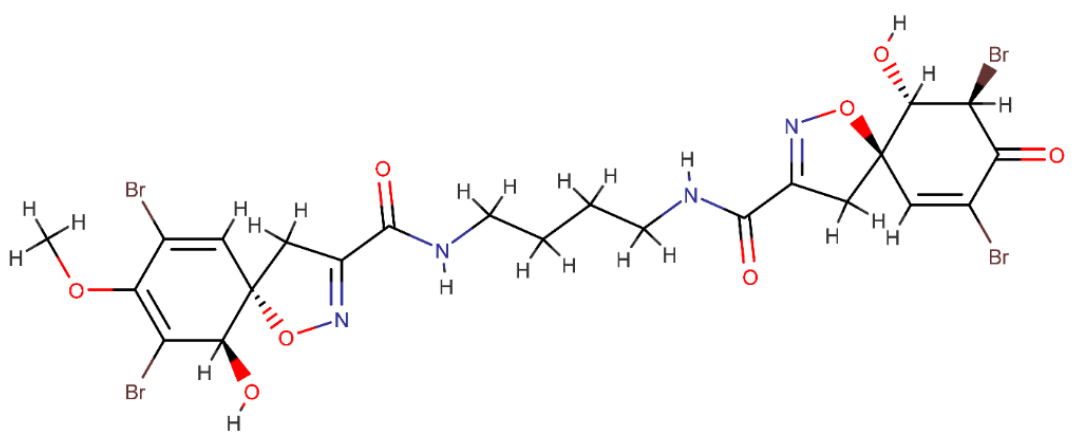<smiles>Cc1ccc(S(=O)(=O)/N=C2/NC(=O)/C(=C/c3ccc(C)o3)S2)cc1</smiles>

Figure 5. 2d structure of co-crystalized ligand.

\subsection{Discussion.}

HCV NS5B polymerase serves as a target enzyme for antiviral therapy against HCV $[9,10]$ as it plays a vital role in the replication and infection of the HCV virus [7].

This study tends to predict the best inhibitor for HCV NS5B from the alkaloid library to inhibit its action using a molecular docking approach.

The molecular docking scores result shown in table 1 shows that 3-(4-methoxyphenyl)3-[[2-(4-methoxyphenyl)-1-oxoethyl]amino]propanoic acid, 14-norpseurotin A, and (+)aplysinilin is predicted to have a better inhibitory effect on HCV NS5B RNA polymerase when compared to the co-crystallized ligand with docking score of $-8.567 \mathrm{Kcal} / \mathrm{mol},-7.233 \mathrm{Kcal} / \mathrm{mol}$ and $-7.173 \mathrm{Kcal} / \mathrm{mol}$ respectively, compared to the co-crystallized ligand with docking score of $-6.429 \mathrm{Kcal} / \mathrm{mol}$. A bar chart representation of the result is shown in Figure 11, which shows the pictorial differences between the hit compound's docking score compared with the target protein co-crystalized ligand.

This in silico study shows that all three lead compounds have relatively lesser binding energy than the co-crystallized ligand; this predicts that these compounds may be considered good inhibitors of HCV NS5B polymerase. 
However, 3-(4-methoxyphenyl)-3- [[2-(4-methoxyphenyl)- 1-oxoethyl]amino] propanoic acid shows more drug-like characteristics by obeying the rule of five for drug development as shown in Table 1. The rule of five is valid when $\mathrm{MW}<500, \mathrm{QPlogPo} / \mathrm{w}<5$, donor $\mathrm{HB} \leq 5$, acceptor $\mathrm{HB} \leq 10$. HOA: Human Oral Absorption. The values 1, 2, and 3 for low, medium, and high, respectively [33], which therefore predicts that 3-(4-methoxyphenyl)3-[[2-(4-methoxyphenyl)-1-oxoethyl]amino]propanoic acid may be a good drug molecule that may inhibit HCV NS5B.

Structural-based drug design is solemnly based on protein-ligand interaction [34]. Typical research work like this involves a situation whereby HCV NS5B inhibition is desired, and relevant inhibition is decided by interactions with some amino acid residues at the enzyme's active site within $4 \AA \hat{\text { }}$ hydrogen bonding distance [34]. These amino acid residues play a significant role in forming the Protein-ligand complex formed by HCV NS5B and either of the compounds involved in this research work.

The $2 \mathrm{~d}$ and $3 \mathrm{~d}$ interaction of this work shows: that 3-(4-methoxyphenyl)-3-[[2-(4methoxyphenyl)-1-oxoethyl]amino]propanoic acid has hydrogen bonds with LYS 533 and SER 476, a salt bridge with ARG 501 and Pi-cations with ARG 501. 14-norpseurotin A has a hydrogen bond with SER 476, salt bridges with LYS 533 and ARG 501, and Pi-Pi stacking with TRP 528. (+)-aplysinilin has hydrogen bonds with LEU 497 and ARG 490 and salt bridges with ARG 498 and ARG 501. While the co-crystallized ligand, on the other hand, the cocrystallized ligand has only hydrogen bonds with TYR 477 and ARG 501 as shown in figures $6,7,8$, and 9, respectively, in 3D format and shown in figure 10 in 2D representation.

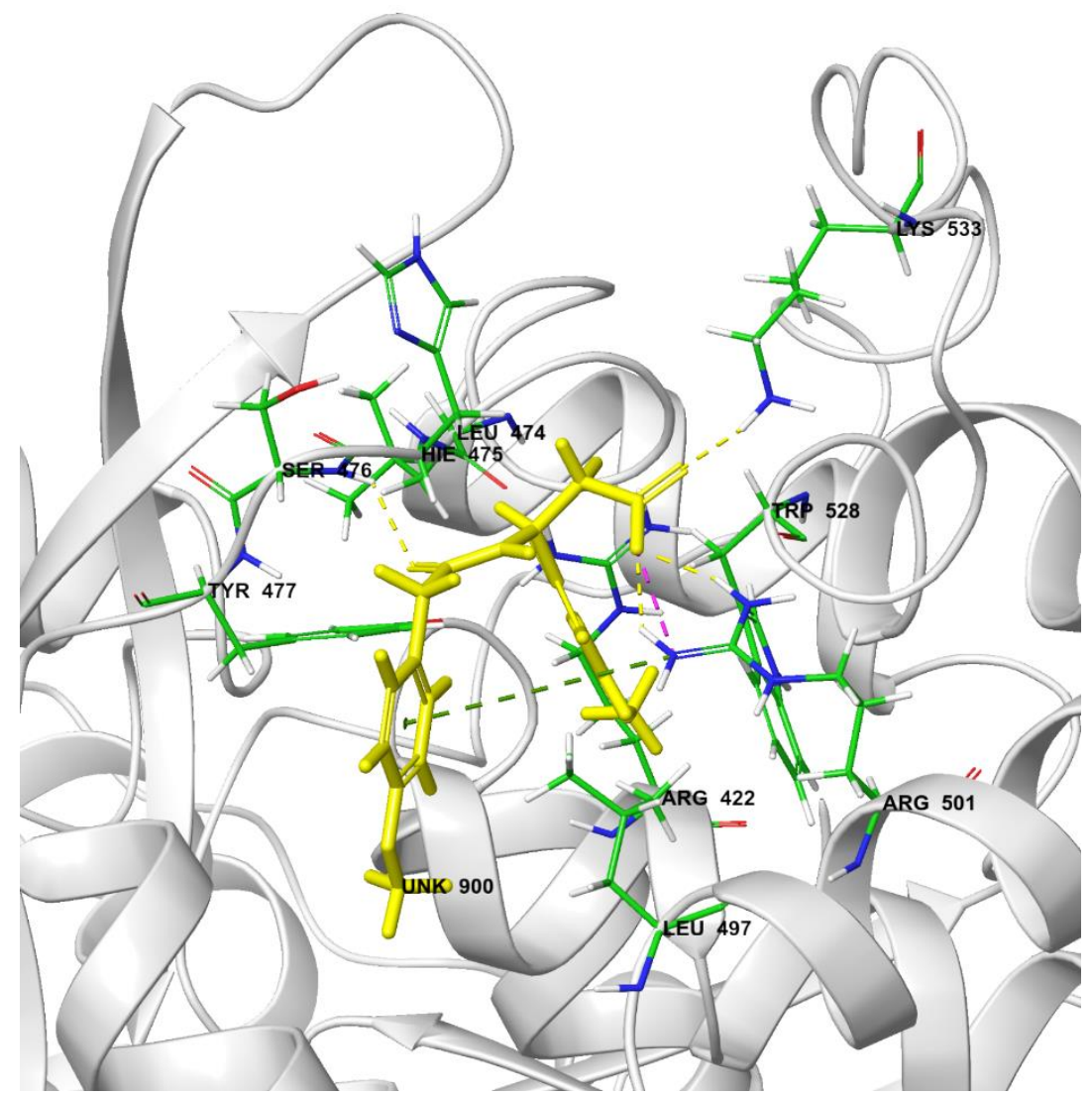

Figure 6. The binding pose analysis of 3-(4-methoxyphenyl)-3-[[2-(4-methoxyphenyl)-1oxoethyl]amino]propanoic acid to the active site of NS5B. 


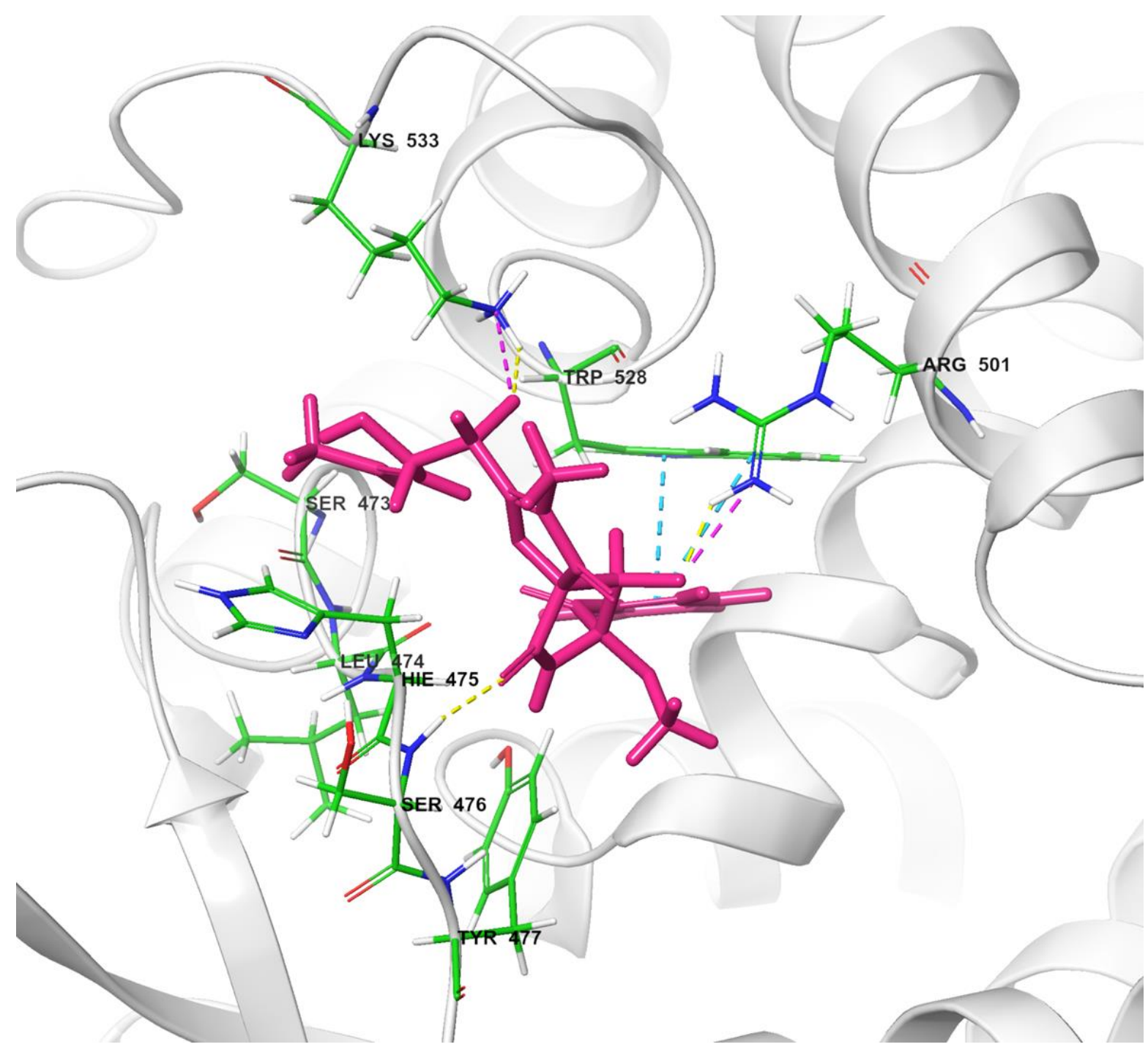

Figure 7. The binding pose analysis of 14-norspeurotin A to the active site of NS5B.

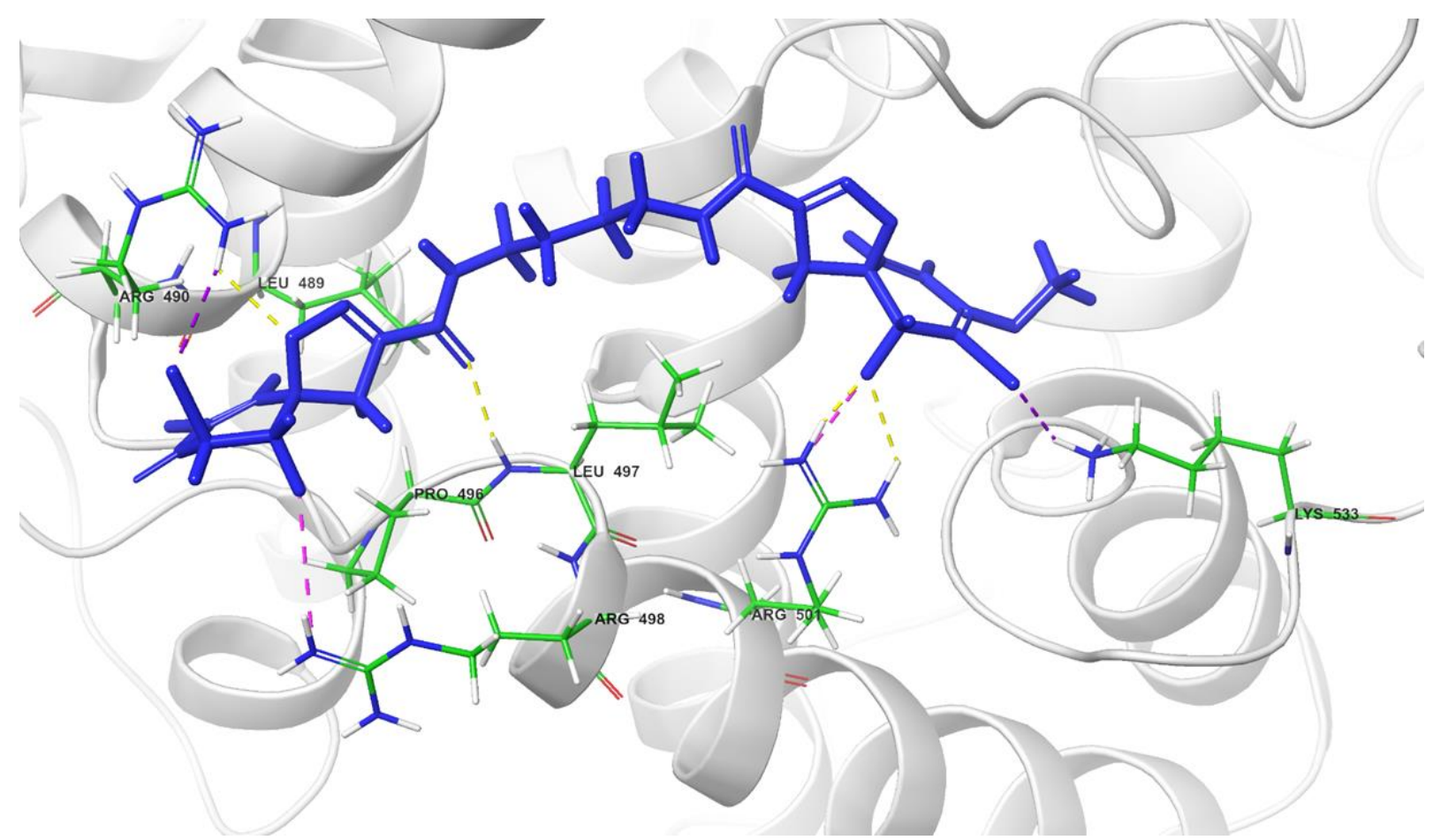

Figure 8. The binding pose analysis of (+)-aplysinilin to the active site of NS5B. 


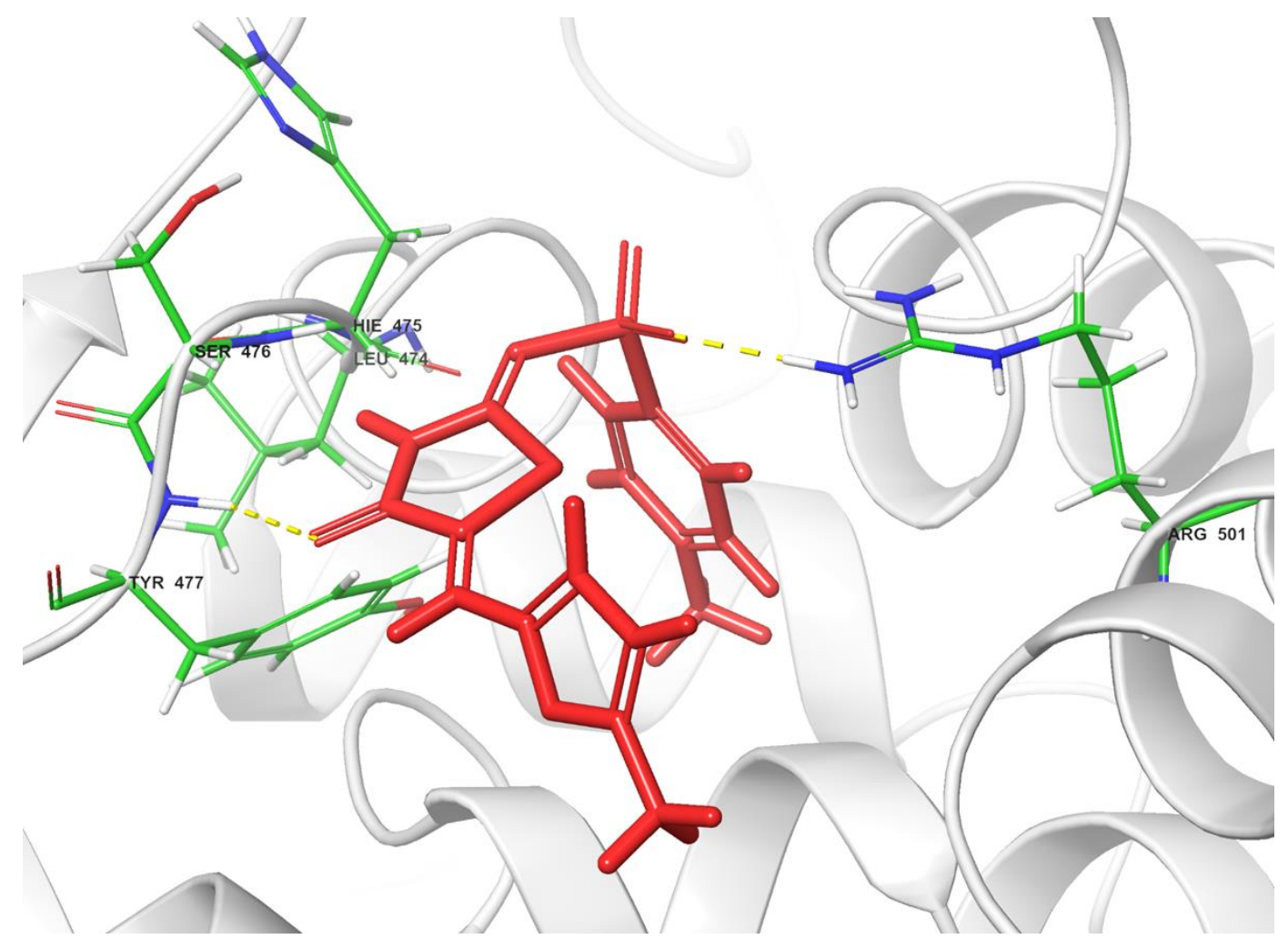

Figure 9. The binding pose analysis of co-crystallized ligand to the active site of NS5B.

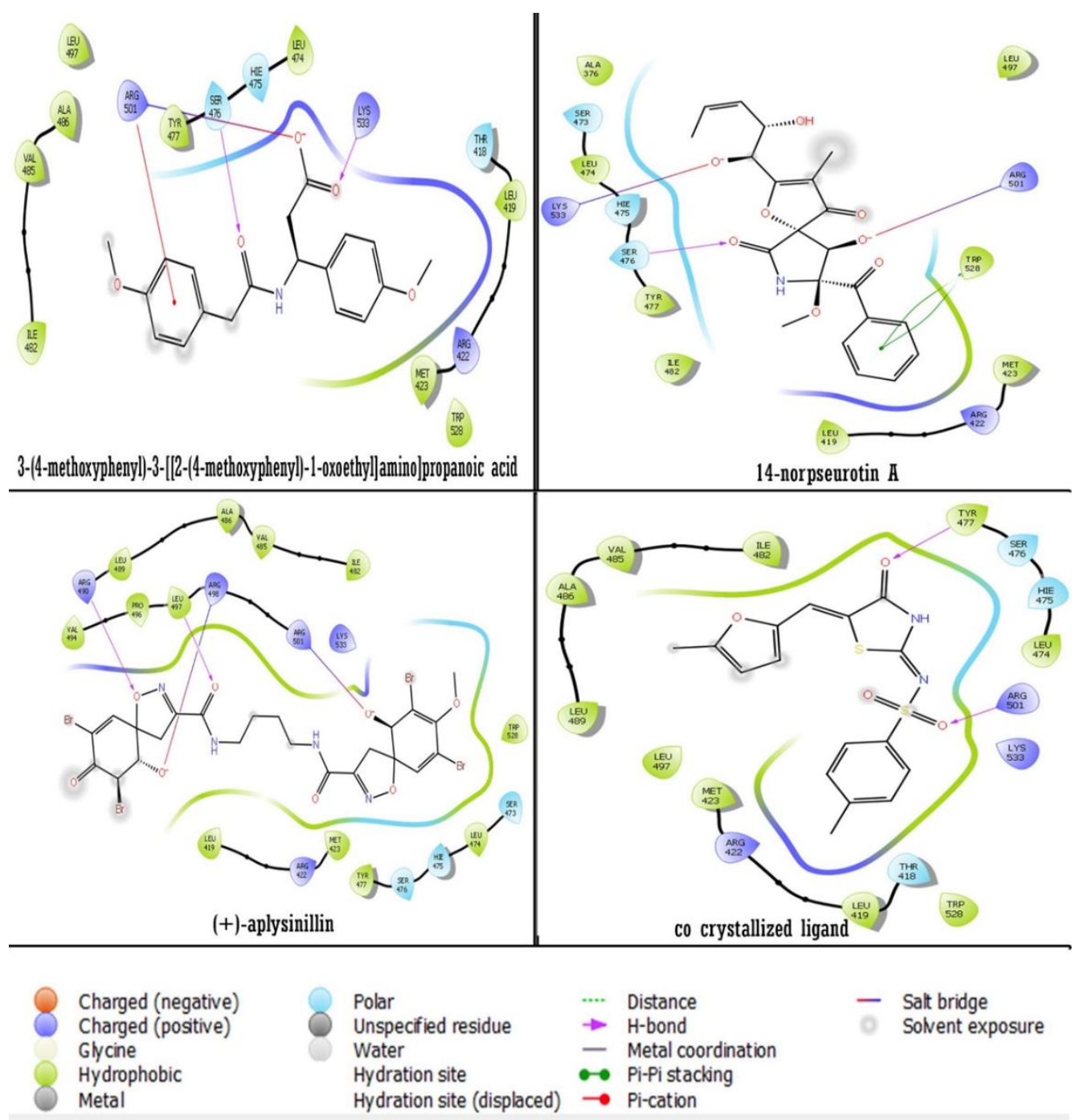

Figure 10. 2d interaction diagram if hit compounds abd co ligand to the active site of NS5B. 
Table 1. Result from Docking and ADME/Tox screening.

\begin{tabular}{l|c|c|c|c|c|c|c} 
Compounds & $\begin{array}{c}\text { Docking } \\
\text { Score }\end{array}$ & mol MW & donorHB & accptHB & QPlogPo/w & $\begin{array}{c}\text { Percent } \\
\text { Human } \\
\text { Oral Absorption }\end{array}$ & $\begin{array}{c}\text { Human } \\
\text { oral } \\
\text { Absorption }\end{array}$ \\
\hline Co ligand & 6.492 & 362.418 & 0 & 7 & 1.971 & 80.436 & 0 \\
\hline $\begin{array}{l}\text { 3-(4-methoxyphenyl)-3- } \\
\text { [[2-(4-methoxyphenyl)-1- } \\
\text { oxoethyl]amino]propanoic } \\
\text { acid }\end{array}$ & -8.567 & 343.379 & 1 & 5 & 3.419 & 82.187 & 3 \\
\hline 14-norpseurotin A & -7.233 & 417.415 & 2 & 11.1 & 0.767 & 64.622 & 3 \\
\hline (+)-aplysinillin & -7.173 & 804.081 & 4 & 14.65 & 2.517 & 36.922 & 1
\end{tabular}

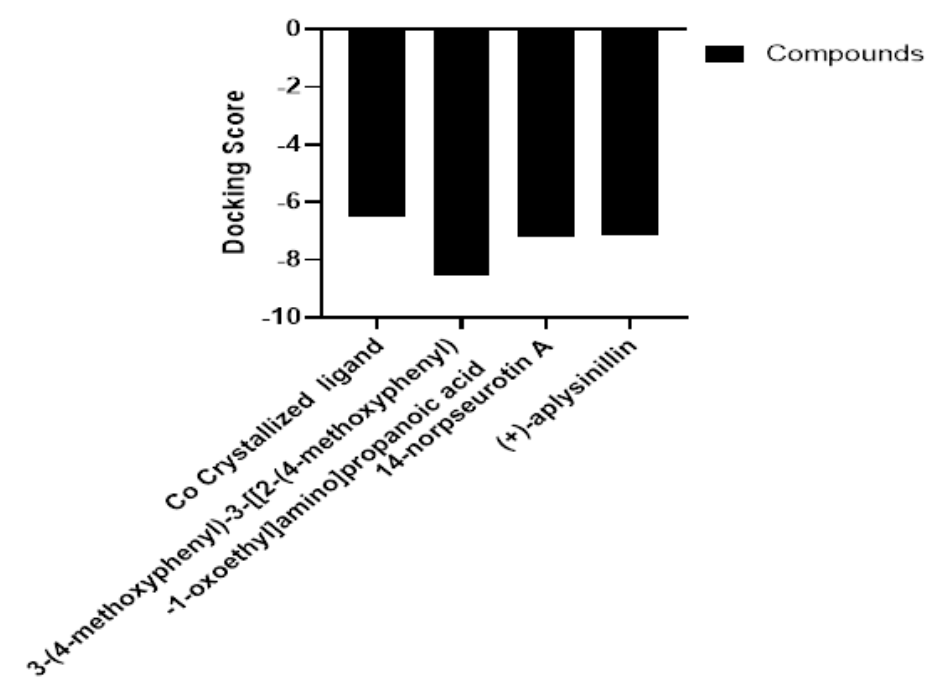

Figure 11. Bar chart showing the pictorial representation of the differences between the docking scores of hit compounds compared to the co-crystalized ligand.

\section{Conclusions}

After the whole experiment, it was showed that 3-(4-methoxyphenyl)-3-[[2-(4methoxyphenyl)-1-oxoethyl]amino]propanoic acid, 14-norpseurotin A, and (+)-aplysinilin are predicted to be suitable inhibitors for NS5B and however, 3-(4-methoxyphenyl)-3-[[2-(4methoxyphenyl)-1-oxoethyl]amino]propanoic acid shows more drug-like characteristics that other compounds. The interactions displayed by these compounds show clearly that these three hit compounds have better inhibitory potential against HCV NS5B. And hence are predicted to be promising drug molecules that might lead to a pharmacological solution to HCV; however, further in vivo and in vitro analyses are recommended for further validation of this experiment.

\section{Funding}

This research received no external funding.

\section{Acknowledgments}

This research has no acknowledgment.

\section{Conflicts of Interest}

The authors declare no conflict of interest. 


\section{References}

1. Lauer, G.M.; Walker, B.D. Hepatitis C Virus Infection. New England Journal of Medicine 2001, 345, 41-52, https://doi.org/10.1056/NEJM200107053450107.

2. Fattovich, G.; Stroffolini, T.; Zagni, I.; Donato, F. Hepatocellular carcinoma in cirrhosis: Incidence and risk factors. Gastroenterology 2004, 127, S35-S50, https://doi.org/10.1053/j.gastro.2004.09.014.

3. Parkin, D.M. The global health burden of infection-associated cancers in the year 2002. International Journal of Cancer 2006, 118, 3030-3044, https://doi.org/10.1002/ijc.21731.

4. Lee, M.-H.; Yang, H.-I.; Yuan, Y.; L'Italien, G.; Chen, C.-J. Epidemiology and natural history of hepatitis C virus infection. World J Gastroenterol 2014, 20, 9270-9280.

5. Graf, C.; Mücke, M.M.; Dultz, G.; Peiffer, K.-H.; Kubesch, A.; Ingiliz, P.; Zeuzem, S.; Herrmann, E.; Vermehren, J. Efficacy of Direct-acting Antivirals for Chronic Hepatitis C Virus Infection in People Who Inject Drugs or Receive Opioid Substitution Therapy: A Systematic Review and Meta-analysis. Clinical Infectious Diseases 2020, 70, 2355-2365, https://doi.org/10.1093/cid/ciz696.

6. Hopkins, L.; Dunlap, T.; Cline, H. Pharmacology Update for the Treatment of Hepatitis C Virus. Nursing Clinics of North America 2020, 55, 347-359, https://doi.org/10.1016/j.cnur.2020.06.008.

7. Kolykhalov Alexander, A.; Mihalik, K.; Feinstone Stephen, M.; Rice Charles, M. Hepatitis C Virus-Encoded Enzymatic Activities and Conserved RNA Elements in the 3' Nontranslated Region Are Essential for Virus Replication In Vivo. Journal of Virology 2000, 74, 2046-2051, https://doi.org/10.1128/JVI.74.4.20462051.2000.

8. Khalid, H.; Landry, K.B.; Ijaz, B.; Ashfaq, U.A.; Ahmed, M.; Kanwal, A.; Froeyen, M.; Mirza, M.U. Discovery of novel Hepatitis C virus inhibitor targeting multiple allosteric sites of NS5B polymerase. Infection, Genetics and Evolution 2020, 84, https://doi.org/10.1016/j.meegid.2020.104371.

9. De Clercq, E.; Holý, A. Strategies in the design of antiviral drugs. Nat Rev Drug Discov. 2002, 1, 13-25, https://doi.org/10.1038/nrd703.

10. Jim Zhen, W.; Zhi, H. Targeting NS5B RNA-Dependent RNA Polymerase for Anti-HCV Chemotherapy. Current Drug Targets - Infectious Disorders 2003, 3, 207-219, https://doi.org/10.2174/1568005033481114.

11. Ellis, D.A.; Blazel, J.K.; Webber, S.E.; Tran, C.V.; Dragovich, P.S.; Sun, Z.; Ruebsam, F.; McGuire, H.M.; Xiang, A.X.; Zhao, J.; Li, L.-S.; Zhou, Y.; Han, Q.; Kissinger, C.R.; Showalter, R.E.; Lardy, M.; Shah, A.M.; Tsan, M.; Patel, R.; LeBrun, L.A.; Kamran, R.; Bartkowski, D.M.; Nolan, T.G.; Norris, D.A.; Sergeeva,

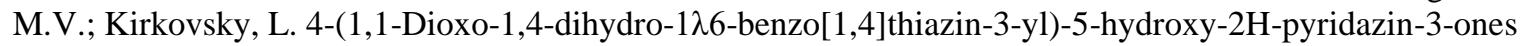
as potent inhibitors of HCV NS5B polymerase. Bioorganic \& Medicinal Chemistry Letters 2008, 18, 46284632, https://doi.org/10.1016/j.bmcl.2008.07.014.

12. Pelletier, S.W. The nature and definition of an alkaloid. Alkaloids: chemical and biological perspectives. 1983, $1,1-31$.

13. Acamovic, T.; Stewart, C.S.; Pennycott, T.W. eds. Poisonous plants and related toxins. 2004.

14. Debnath, B.; Singh, W.S,; Das, M.; Goswami, S.; Singh, M.K.; Maiti, D.; Manna, K. Role of plant alkaloids on human health: A review of biological activities. Mater. Today Chem. 2018, 9, 56-72, https://doi.org/10.1016/j.mtchem.2018.05.001.

15. Roy, A. A review on the alkaloids an important therapeutic compound from plants. IJPB. 2017, 3, 1-9.

16. Mondal, A.; Gandhi, A.; Fimognari, C.; Atanasov, A.G.; Bishayee, A. Alkaloids for cancer prevention and therapy: Current progress and future perspectives. Eur J Pharmacol. 2019, 858, https://doi.org/10.1016/j.ejphar.2019.172472.

17. Liu, C.; Yang, S.; Wang, K.; Bao, X.; Liu, Y.; Zhou, S.; Liu, H.; Qiu, Y.; Wang, T.; Yu, H. Alkaloids from Traditional Chinese Medicine against hepatocellular carcinoma. Biomedicine \& Pharmacotherapy 2019, 120, https://doi.org/10.1016/j.biopha.2019.109543.

18. Rasouli, H.; Yarani, R.; Pociot, F.; Popović-Djordjević, J. Anti-diabetic potential of plant alkaloids: Revisiting current findings and future perspectives. Pharmacological Research 2020, 155, https://doi.org/10.1016/j.phrs.2020.104723.

19. Pagadala, N.S.; Syed, K.; Tuszynski, J. Software for molecular docking: a review. Biophysical Reviews 2017, 9, 91-102, https://doi.org/10.1007/s12551-016-0247-1.

20. Tao, X.; Huang, Y.; Wang, C.; Chen, F.; Yang, L.; Ling, L.; Che, Z.; Chen, X. Recent developments in molecular docking technology applied in food science: a review. International Journal of Food Science \& Technology 2020, 55, 33-45, https://doi.org/10.1111/ijfs.14325.

21. Kim, S.; Thiessen, P.A.; Bolton, E.E.; Chen, J.; Fu, G.; Gindulyte, A.; Han, L.; He, J.; He, S.; Shoemaker, B.A.; Wang, J.; Yu, B.; Zhang, J.; Bryant, S.H. PubChem Substance and Compound databases. Nucleic acids research 2016, 44, D1202-D1213, https://doi.org/10.1093/nar/gkv951.

22. Release S. 2: LigPrep, Schrödinger, LLC, New York, NY, 2021. https://www.schrodinger.com/citations

23. Shelley, J.C.; Cholleti, A.; Frye, L.L.; Greenwood, J.R.; Timlin, M.R.; Uchimaya, M. Epik: a software program for pKaprediction and protonation state generation for drug-like molecules. Journal of ComputerAided Molecular Design 2007, 21, 681-691, https://doi.org/10.1007/s10822-007-9133-z.

24. Schrödinger Release 2021-1: Epik, Schrödinger, LLC, New York, NY, 2021; https://www.schrodinger.com/citations. 
25. Harder, E.; Damm, W.; Maple, J.; Wu, C.; Reboul, M.; Xiang, J.Y.; Wang, L.; Lupyan, D.; Dahlgren, M.K.; Knight, J.L.; Kaus, J.W.; Cerutti, D.S.; Krilov, G.; Jorgensen, W.L.; Abel, R.; Friesner, R.A. OPLS3: A Force Field Providing Broad Coverage of Drug-like Small Molecules and Proteins. Journal of Chemical Theory and Computation 2016, 12, 281-296, https://doi.org/10.1021/acs.jctc.5b00864.

26. Schrödinger, L.L.C. Schrödinger, LLC. New York, NY: 2017. Schrödinger Suite, 2, 2017-1, 2017; https://www.schrodinger.com/citations.

27. Asthana, S.; Agarwal, T.; Banerjee, I.; Ray, S.S. In Silico screening to elucidate the therapeutic potentials of Asparagamine A. International Journal of Pharmacy and Pharmaceutical Sciences 2014, 6, 247-253.

28. Burley, S.K.; Berman, H.M.; Bhikadiya, C.; Bi, C.; Chen, L.; Di Costanzo, L.; Christie, C.; Dalenberg, K.; Duarte, J.M.; Dutta, S.; Feng, Z.; Ghosh, S.; Goodsell, D.S.; Green, R.K.; Guranović, V.; Guzenko, D.; Hudson, B.P.; Kalro, T.; Liang, Y.; Lowe, R.; Namkoong, H.; Peisach, E.; Periskova, I.; Prlić, A.; Randle, C.; Rose, A.; Rose, P.; Sala, R.; Sekharan, M.; Shao, C.; Tan, L.; Tao, Y.-P.; Valasatava, Y.; Voigt, M.; Westbrook, J.; Woo, J.; Yang, H.; Young, J.; Zhuravleva, M.; Zardecki, C. RCSB Protein Data Bank: biological macromolecular structures enabling research and education in fundamental biology, biomedicine, biotechnology and energy. Nucleic acids research 2019, 47, D464-D474, https://doi.org/10.1093/nar/gky1004.

29. David, T.I.; Adelakun, N.S.; Omotuyi, O.I.; Metibemu, D.S.; Ekun, O.E. Molecular docking analysis of phyto-constituents from Cannabis sativa with pfDHFR. Bioinformation. 2018, 14, 574-579, https://doi.org/10.6026/97320630014574.

30. Friesner, R.A.; Banks, J.L.; Murphy, R.B.; Halgren, T.A.; Klicic, J.J.; Mainz, D.T.; Repasky, M.P.; Knoll, E.H.; Shelley, M.; Perry, J.K.; Shaw, D.E.; Francis, P.; Shenkin, P.S. Glide: A New Approach for Rapid, Accurate Docking and Scoring. 1. Method and Assessment of Docking Accuracy. Journal of Medicinal Chemistry 2004, 47, 1739-1749, https://doi.org/10.1021/jm0306430.

31. Schrödinger Release 2021-1: Glide, Schrödinger, LLC, New York, NY, 2021; https://www.schrodinger.com/citations.

32. Release, S. 2: QikProp, Schrödinger. 2021; https://www.schrodinger.com/citations.

33. Lipinski, C.A. Lead- and drug-like compounds: the rule-of-five revolution. Drug Discovery Today: Technologies 2004, 1, 337-341, https://doi.org/10.1016/j.ddtec.2004.11.007.

34. Inyang, O.K.; Omotuyi, O.I.; Ogunleye, A.J.; Eniafe, G.O.; Adewumi, B. Molecular Interaction and Inhibitory Potential of Polyphenol on DNA Repair Pathway in Small Cell Lung Cancer: A Computational Study. J Anal Pharm Res. 2017, 6, https://doi.org/10.15406/japlr.2017.06.00178. 